\title{
Characterization of the Asiatic Acid Glucosyltransferase, UGT73AH1, Involved in Asiaticoside Biosynthesis in Centella asiatica (L.) Urban
}

\author{
Ok Tae Kim ${ }^{1, *}$ (D), Mei Lan Jin ${ }^{1}$, Dae Young Lee ${ }^{1}$ (D) and Reinhard Jetter ${ }^{2,3}$ \\ 1 Department of Herbal Research, National Institute of Horticultural and Herbal Science, RDA, \\ Eumseong 27709, Korea; milan80623@korea.kr (M.L.J.); dylee0809@korea.kr (D.Y.L.) \\ 2 Department of Botany, University of British Columbia, 6270 University Blvd., \\ Vancouver, BC V6T 1Z4, Canada; Reinhard.jetter@botany.ubc.ca \\ 3 Department of Chemistry, University of British Columbia, 2036 Main Mall, \\ Vancouver, BC V6T 1Z1, Canada \\ * Correspondence: kimot99@hanmail.net; Tel.: +82-63-238-6131; Fax: +82-63-238-6105
}

Received: 13 November 2017; Accepted: 1 December 2017; Published: 6 December 2017

\begin{abstract}
Centella asiatica (L.) Urban contains two ursane-type triterpene saponins, asiaticoside and madecassoside, as major secondary metabolites. In order to select candidate genes encoding UDP-glucosyltransferases (UGTs) involved in asiaticoside biosynthesis, we performed transcriptomic analysis of leaves elicited by methyl jasmonate (MeJA). Among the unigenes, 120 isotigs and 13 singletons of unique sequences were annotated as UGTs, including 37 putative full-length cDNAs, and 15 of the putative UGT genes were named according to the UGT committee nomenclature protocols. One of them, UGT73AH1, was characterized by heterologous expression in Escherichia coli BL21 (DE3) cells. After induction with IPTG, a total protein extract was assayed with UDP-glucose and asiatic acid. UPLC-QTOF/MS analysis showed that UGT73AH1 catalyzes the glycosylation of asiatic acid to its monoglucoside. It remains unclear whether glycosylation occurs on the triterpene C- $2 \alpha, \mathrm{C}-3 \beta, \mathrm{C}-23$, or C-28 position. However, it is very likely that UGT73AH1 glucosylates the C-28 position, because only C-28 bears a glucose moiety in the final pathway product of asiatic acid, while $\mathrm{C}-2 \alpha, \mathrm{C}-3 \beta$, and C-23 remain un-conjugated.
\end{abstract}

Keywords: Centella asiatica; transcriptome; triterpene saponins; UDP-glycosyltransferase

\section{Introduction}

Many plant secondary metabolites have characteristic carbon skeletons linked to one or more sugar moieties, and such glycosylation strongly affects the solubility, biochemical properties, subcellular localization, and biological function of the natural products [1]. Typically, glycosylation occurs only after the carbon skeletons of the aglycones have been established, in the final steps of the natural product biosynthesis pathways. Respective glycosylation reactions are generally catalyzed by transferases using activated sugar substrates, belonging to the enzyme family of UDP-glycosyltransferases (UGTs) [2].

Plants produce a large variety of triterpene saponins, with molecular structures featuring an alicyclic triterpene backbone linked through glucosidic bonds to one or more sugar moieties [3]. More than 300 different triterpenoid carbon skeletons have been described, and they can carry diverse glycosyl side chains on one or more oxygen functionalities [4-6]. Many plant species contain triterpene saponins derived from oleanane-type triterpenoids, such as glycyrrhizin from licorice, saikosaponins from Bupleurum falcatum, and soyasaponins from Glycine max. 
The functional characterization of UGT genes involved in triterpenoid biosynthesis has seen relatively little progress over the last decades, likely because all plant genomes encode numerous UGTs homologs, making it difficult to select and characterize candidate genes [7]. For example, the Arabidopsis thaliana and G. max genomes contain 115 and 242 UGT genes, respectively [8-11]. Recently, transcriptome sequencing has emerged as a tool to identify candidate UGT genes involved in the biosynthesis of particular triterpene saponins. In particular, the UGT73K1 and UGT71G1 genes from Medicago truncatula, UGT73P2 and UGT91H4 from G. max, UGT74M1 from Saponaria vaccaria, UGT73C10 and UGT73C11 from Barbarea vulgaris, and UGT74H6 from Avena strigosa, were shown to be involved in glycosylation of oleanane saponins $[9,12-16]$. Thus, enzymes belonging to the UGT subfamilies 71 to 74 appear able to link a glucose to the $\mathrm{C}-3 \beta$ or $\mathrm{C}-28$ functionalities of oleanane structures, implying that highly conserved motifs may convey some product specificity for triterpene glycosylation. However, only one gene, UGT73AD1, encoding a UGT responsible for forming ursane-type triterpene saponins has been identified to date [17], and it hence remains to be determined whether UGT71-74 homologs may also accept this different type of aglycone substrate.

The medicinal plant Centella asiatica (L.) Urban contains mainly ursane-type triterpene saponins, the most prominent ones being madecassoside and asiaticoside [18]. Both madecassoside and asiaticoside accumulate to relatively high concentrations in the leaves of $C$. asiatica, which have traditionally been used in various pharmaceutical applications. It is now firmly established that the major bioactivities of $C$. asiatica leaf extracts are due to these saponins, including memory improvement, wound and vein healing, antihistaminic, antiulcer and antilepsory treatments, as an antidepressant, and as antibacterial, antifungal, and antioxidant agents [19-21].

Asiaticoside and madecassoside have identical sugar chains (glucose-glucose-rhamnose) bonded to their carboxyl groups [22]. Therefore, it has been hypothesized that both compounds are formed by glycosylation of asiatic acid and madecassic acid, respectively, probably catalyzed by UDP-glucosyltransferases (UGTs) that first link a glucose to the C-28 carboxyl group. A further glycosylation and a consecutive rhamnosylation reaction then likely lead to the final products (Figure 1). Recently, de Costa et al. [17] reported that UGT73AD1 plays a role in linking a glucose at a carboxyl moiety in C. asiatica. However, other UGT genes have not been characterized, to date.

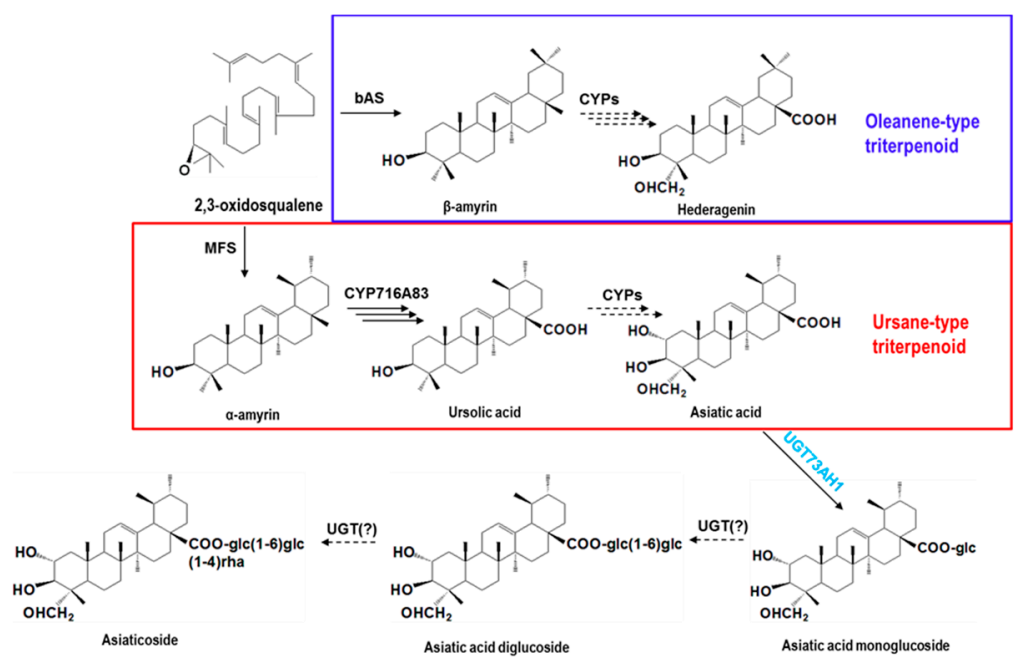

Figure 1. Putative biosynthetic pathway leading to triterpene saponins in Centella asiatica. The precursors, $\beta$-amyrin and $\alpha$-amyrin, are converted by a series of oxidative reactions to oleaneneand ursane-type aglycones, respectively. In the ursane-type triterpenoid pathway, asiatic acid is converted by UDP-glucosyltransferases (UGTs) to asiaticoside. The following enzymes are represented: bAS, $\beta$-amyrin synthase; MFS, multifunctional triterpene synthase; CYP716A83, C-28 oxidase; CYPs, cytochrome P450 monooxygenases; UGT73AH1, 28-O-glucosytransferase. 
Therefore, the goal of the present study was to isolate and characterize the glycosyl transferase(s) responsible for the formation of asiaticoside triterpene saponins in C. asiatica. To this end, we selected UGT candidate genes from transcriptomic data of elicited C. asiatica leaves, and characterized them by heterologous expression.

\section{Results and Discussion}

The goal of the present study was to identify one or more glycosyl transferases involved in asiaticoside formation using an omics approach. In particular, the transcriptome of MeJA-elicited C. asiatica leaves was analyzed, glycosyltransferase unigenes were selected based on phyologeny and expression patterns, and the resulting candidate genes were biochemically characterized using heterologous expression in Escherichia coli and product analysis with UPLC-QTOF/MS.

\subsection{Transcriptome Analysis of Leaves Elicited with MeJA}

Transcriptome analysis of elicited C. asiatica leaves yielded a total of 1,282,298 raw reads of $415.4 \mathrm{bps}$ on average. After deleting adaptor sequences and eliminating reads $<100 \mathrm{bps}, 40,064$ unique sequences comprising 31,050 isotigs and 9014 singletons were identified and annotated, based on sequence similarity with complete sequences in the Swiss-Prot, TAIR (The Arabidopsis Information Resource), NCBI non-redundant protein (Nr), and NCBI non-redundant nucleotide (Nt) databases (Table 1). A total of 25,978 unigenes had significant matches in the databases. Overall, $64.8 \%$ of the unique sequences were annotated from all databases under highly stringent conditions (cutoff $=10^{-3}$ ).

Table 1. Summary of the annotation rates of $C$. asiatica unique sequences in various public database.

\begin{tabular}{ccc}
\hline The Public Database & No. of Unique Sequences & Annotation Percentage (\%) \\
\hline TAIR & 14,051 & 35.0 \\
Swiss-Prot & 15,171 & 37.9 \\
Nr & 17,807 & 44.4 \\
Nt & 16,709 & 41.7 \\
Total & 25,978 & 64.8 \\
\hline
\end{tabular}

TAIR = The Arabidopsis Information Resource; $\mathrm{Nr}=\mathrm{NCBI}$ non-redundant protein; $\mathrm{Nt}=\mathrm{NCBI}$ non-redundant nucleotide.

\subsection{Selection of Candidate UGT Genes for Asiaticoside Biosynthesis}

The $C$. asiatica transcriptome dataset contained 133 unique sequences annotated as UGTs based on BLAST similarity, including 120 isotigs and 13 singletons, and 37 putative full-length cDNAs (Table S1). In comparison, Sangwan et al. [23] had reported 169 unique UGT sequences based on transcriptome analysis of $C$. asiatica leaves using Hiseq data, however, without providing information on gene names. Of the 37 UGTs identified here, 15 were named according to the UGT nomenclature rules [24], while the remaining 22 sequences could not be assigned as they lacked highly conserved motifs (possibly because they were truncated or incomplete). None of the sequences identified here had significant similarity with the UGT73AD1 gene previously reported to be involved in oxidation of asiatic acid.

Six of the UGT candidate genes identified here belonged to the UGT73 and UGT74 subfamilies previously implicated in triterpene glycosylation. In particular, four of the C. asiatica UGTs (UGT73AH1, UGT73A21, UGT73A22, and UGT73AH2) belonged to the UGT73 subfamily, which comprises the UGT73K1, UGT73P2, and UGT73C10 genes encoding triterpene glucosyltransferases of M. truncatula, G. max, and Barbarea vulgaris, respectively (Figure 2). On the other hand, two C. asiatica UGTs (UGT74AH1 and UGT74AG2) belonged to the UGT74 family, which comprises the UGT74M1 and UGT74H6 genes known to be involved in saponin formation in S. vaccaria and Avena strigosa, respectively. All six C. asiatica UGT73/74 genes had high EST representation and RPKM (Reads per 
Kilobase per Milion mapped reads) values (Table 2), and were therefore considered as good candidates to be involved in asiaticoside formation.

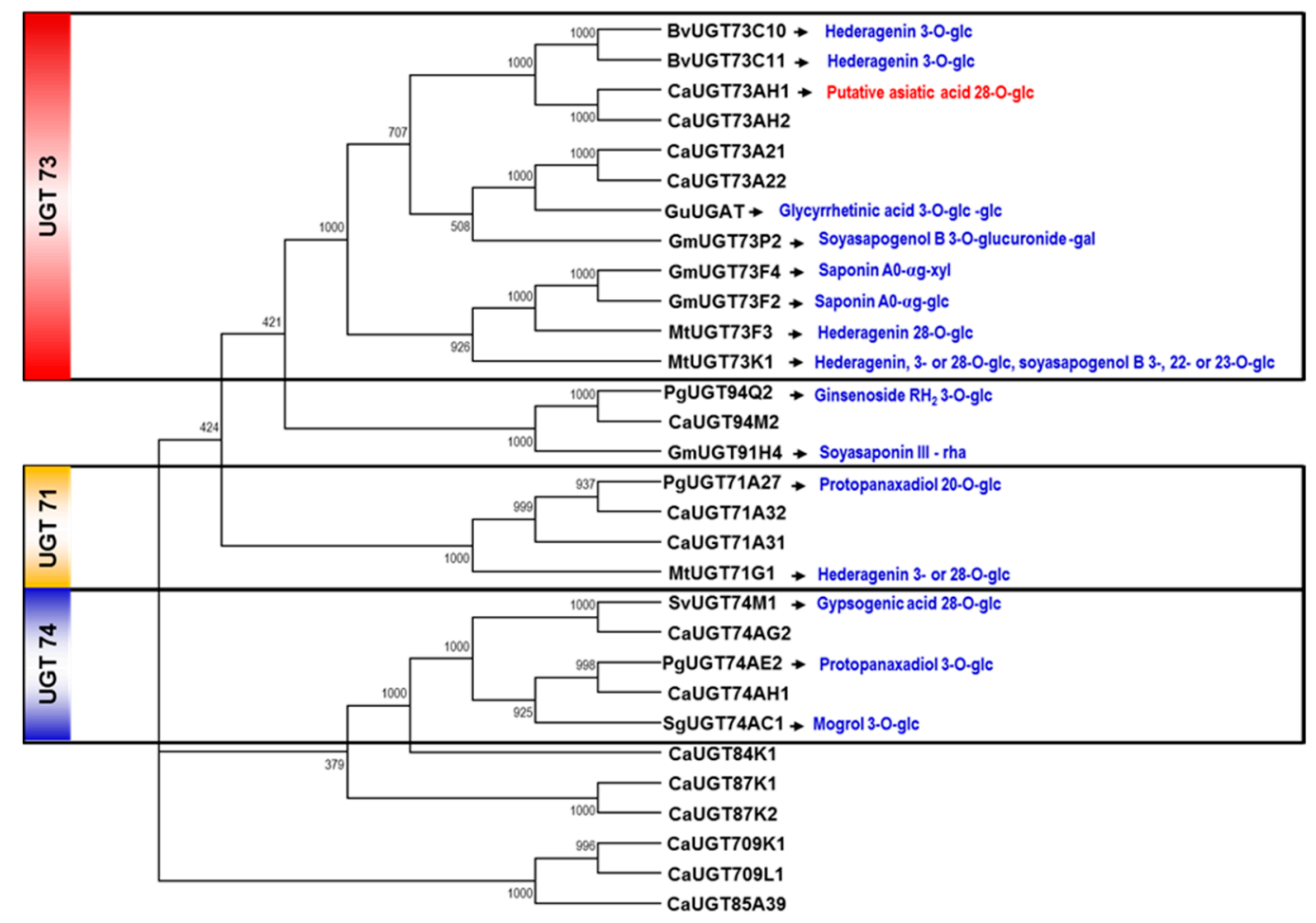

Figure 2. Phylogenetic analysis of UGTs from C. asiatica and other plant species. Sequences of UGTs associated with triterpene saponin biosynthesis were retrieved from the NCBI database: MtUGT71G1 (AAW56092), PgUGT71A27 (KM491309), BvUGT73C10 (AFN26666), BvUGT73C11 (AFN26667), MtUGT73K1 (AAW56091), MtUGT73F3 (ACT34898), GmUGT73F2 (BAM29362), GmUGT73F4 (29363), GmUGT73P2 (BAI99584), GuUGAT (KT759000), SvUGT74M1 (ABK76266), PgUGT74AE2 (JX898529), SvUGT91H4 (BAI99585), and PgUGT94Q2 (JX898530).

Table 2. Selected UGT (UDP-glucosyltransferase) gene candidates resulting from transcriptome analysis of MeJA-elicited C. asiatica leaves.

\begin{tabular}{|c|c|c|c|c|c|}
\hline Gene Name & $\begin{array}{c}\text { Unique } \\
\text { Sequence }\end{array}$ & EST Number & RPKM & Putative Function and Source & $E$-Value \\
\hline UGT73AH1 & Isotig09551 & 235 & 118.01 & $\begin{array}{c}\text { UDP-glycosyltransferase superfamily protein, } \\
\text { putative (Theobroma cacao) }\end{array}$ & 0 \\
\hline UGT73A21 & Isotig08753 & 232 & 101.59 & Glycosyltransferase UGT7 (Bupleurum chinense) & 0 \\
\hline UGT74AH1 & Isotig09014 & 130 & 59.50 & UDP-glycosyltransferase 74E2-like (Vitis vinifera) & 0 \\
\hline UGT73A22 & Isotig09265 & 115 & 55.09 & UDP-glycosyltransferase UGT7 (Bupleurum chinense) & 0 \\
\hline UGT74AG2 & Isotig08223 & 136 & 53.51 & $\begin{array}{l}\text { PREDICTED: UDP-glycosyltransferase 74E1-like } \\
\text { (Solanum lycopersicum) }\end{array}$ & 0 \\
\hline UGT73AH2 & Isotig08281 & 72 & 28.72 & UDP-glycosyltransferase 73C2 (Vitis vinifera) & 0 \\
\hline
\end{tabular}

EST = Expressed sequence tag; RPKM = Reads per kilobase per million mapped reads; UDP = Uridine- $5^{\prime}$-diphosphate.

MeJA is known to strongly stimulate expression of $C$. asiatica genes encoding enzymes along the biosynthetic pathway from farnesyl diphosphate to triterpene glycosides. We therefore hypothesized that UGTs involved in asiaticoside and/or madecassoside formation should also be relatively highly expressed upon MeJA induction. Hence, in order to select strong candidates of UGT, we used qRT-PCR to compare the expression levels of the six UGT candidate genes in C. asiatica hairy root cultures elicited by $0.1 \mathrm{mM}$ MeJA for $24 \mathrm{~h}$ (Figure 3). Transcripts of four UGT genes, UGT73AH1, UGT73A21, UGT73A22, and UGT74AH1, were upregulated after $12 \mathrm{~h}$ of MeJA treatment. In contrast, no significant differences were detected in the expression of the UGT73AH2 gene in response to MeJA treatment, 
and transcripts of UGT74AG2 could be detected neither in control nor in treated hairy roots. Overall, our qRT-PCR results indicated that four genes, UGT73AH1, UGT73A21, UGT73A22, and UGT74AH1, might be involved in asiaticoside biosynthesis.

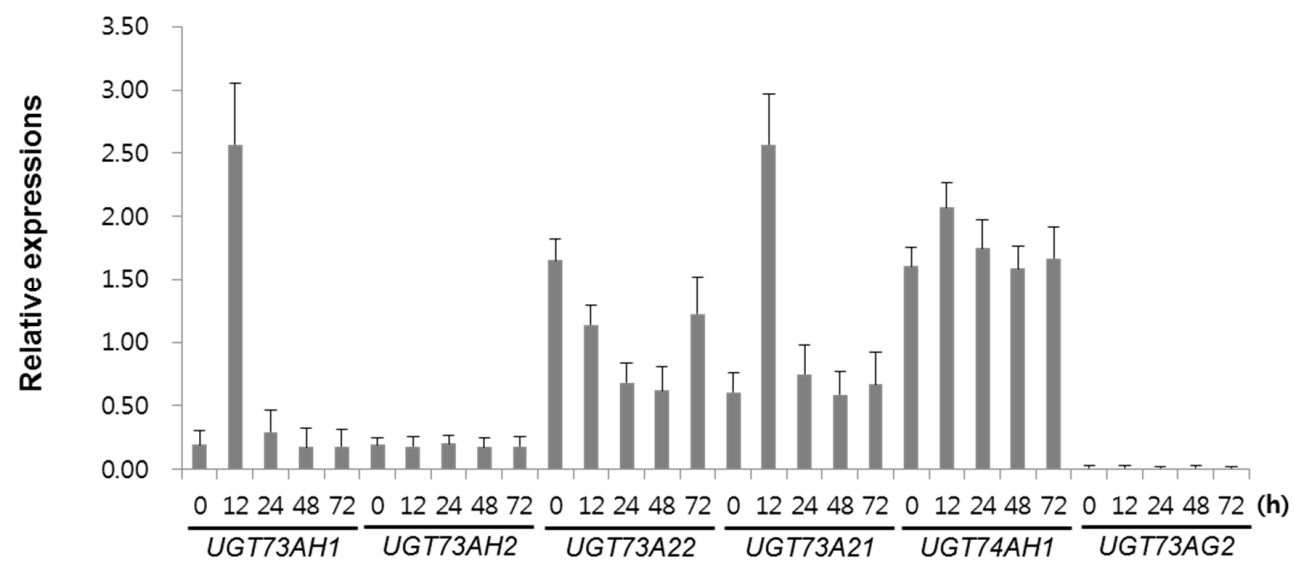

Figure 3. qRT-PCR analysis of UGT expression in hairy roots of $C$. asiatica after treatment with MeJA. The $\beta$-actin gene was used as a reference. The error bars represent standard errors from three biological replicates.

\subsection{Functional Characterization of Candidate UGTs}

The deduced polypeptides of UGT73AH1, UGT73A21, UGT73A22, and UGT74AH1 consist of $501,479,471$ and 462 amino acids, respectively, with amino acid identities between them ranging from $28 \%$ to $40 \%$ (Figure S1). All four C. asiatica UGTs possess the PSPG box, a highly conserved region among UGT enzymes associated with plant secondary metabolism [2].

To test the four candidate genes for UGT activity on triterpene substrates, heterologous expression in E. coli was used. Initially, the four candidate proteins were expressed with His-tags, and SDS-PAGE analysis showed that they accumulated to high levels upon induction (data not shown). However, the proteins were found mainly in inclusion bodies, and all four UGT genes therefore had to be re-cloned into vectors with stop codons to enhance their solubility [25]. The un-tagged proteins were expressed in two E. coli strains, BL21 and BL21 (DE3), however, no soluble protein products were observed for UGT73A21 and UGT73A22 (Figure 4). UGT73AH1 and UGT74AH1 were detected in cell-free extracts by SDS-PAGE analysis, and the two UGT activities were therefore examined further.

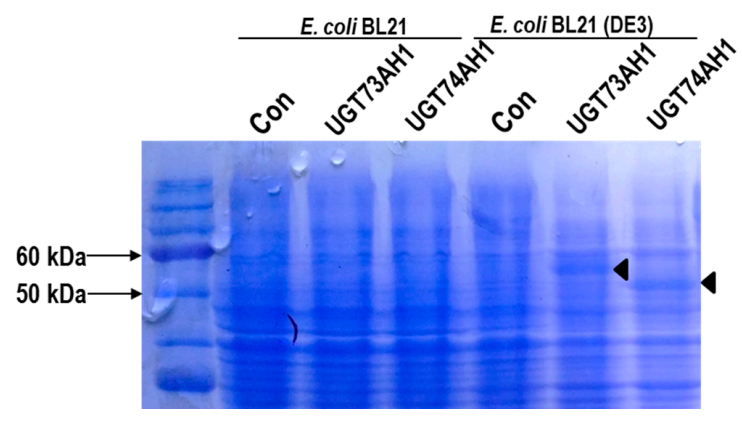

Figure 4. SDS-PAGE analysis of UGT73AH1 and UGT74AH1 heterologously expressed in Escherichia coli. Expression of the proteins in E. coli BL21 and BL21 (DE3) was induced by IPTG for $16 \mathrm{~h}$ at $20^{\circ} \mathrm{C}$.

For biochemical characterization, whole-cell extracts of E. coli expressing the candidate UGTs were assayed in vitro. After incubation of UDP-glucose and asiatic acid as substrates together with either UGT73A21 or UGT73A22 protein, the extracts were analyzed by UPLC-TOF/MS. In the extract from enzymatic mixture with UGT74AH1 protein only, compounds were detected that were also present in 
the control with denatured protein heated at $100^{\circ} \mathrm{C}$ for $10 \mathrm{~min}$, suggesting that this enzyme has no glycosylation activity on asiatic acid. In stark contrast, glycosylation assays with UGT73AH1 resulted in a UPLC peak eluting at 9.2 min under our conditions, which was not detected in the control sample (Figure 5). This compound was unambiguously identified by mass spectra as a mono-glycoside of asiatic acid, with $m / z=673.415$ (Asiatic acid $+\mathrm{Glc}+\mathrm{Na})^{+}$and $m / z 651.453(\text { Asiatic acid }+\mathrm{Glc}+\mathrm{H})^{+}$, reflecting the presence of both a hexose moiety and asiatic acid (Figure 6). Taken together, our results show that UGT73AH1 catalyzes the glycosylation of asiatic acid.

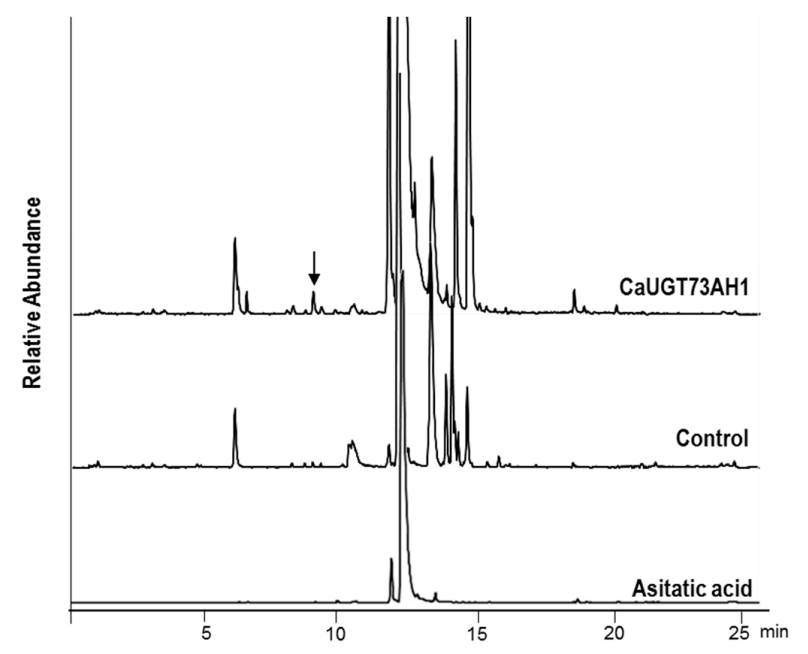

Figure 5. UPLC-QTOF/MS analysis of the products from glycosylation assays of UGT73AH1. Extracts from incubation mixtures containing the protein together with asiatic acid and UDP-glucose are compared with a boiled-protein control and authentic asiatic acid standard. The narrow indicates a peak in the protein incubation extract not present in the control and standard traces. The mass spectral identification of this peak is shown in Figure 6.

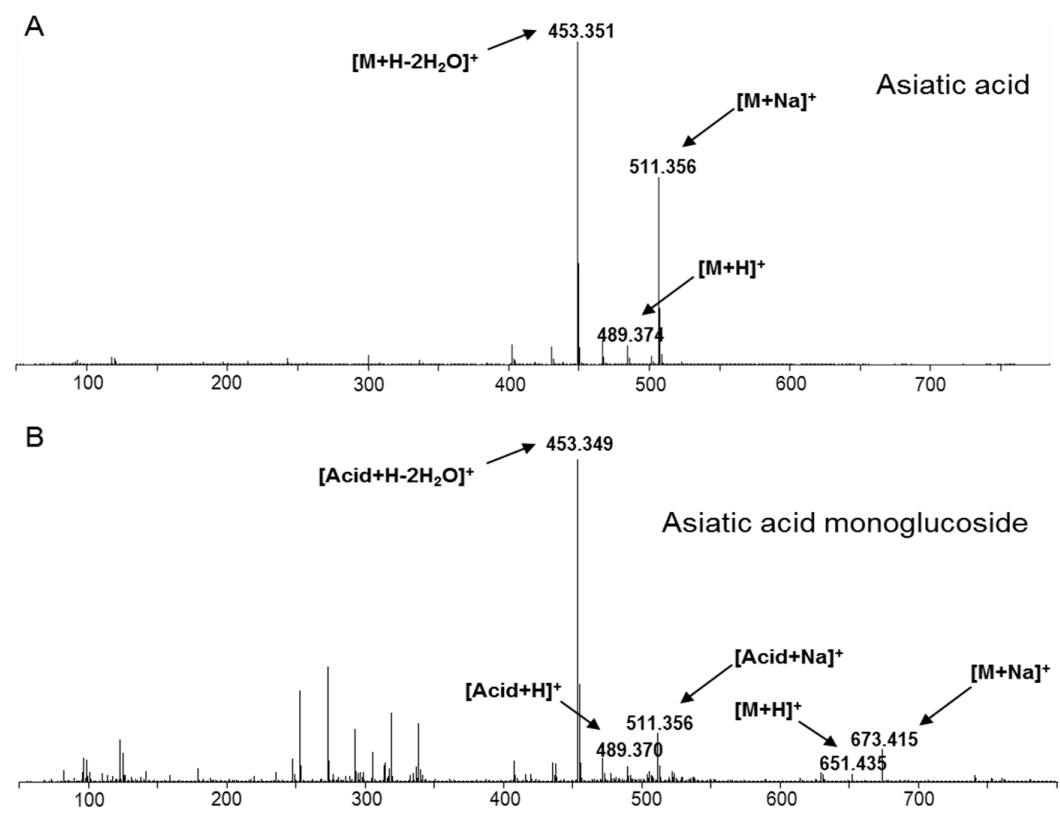

Figure 6. UPLC-QTOF/MS analysis of the products from glycosylation assays of UGT73AH1. (A) Mass spectrum of asiatic acid standard; (B) Mass spectrum of the unique compound formed from enzymatic reaction with UGT73AH1 protein. The fragments $m / z 453,489$, and 511, identical to those of the standard, identify this compound as an asiatic acid derivative. The additional fragments $m / z 651$ and 673 further characterize it as a mono-glycoside. 
Asiatic acid has hydroxyl groups at C-2 $\alpha, \mathrm{C}-3 \beta$, and C-23, as well as a carboxyl moiety at $\mathrm{C}-28$, and thus, four potential glycosylation sites. Unfortunately, our mass spectrometric and chromatographic data alone do not reveal the exact position of the glucose unit in the enzyme assay product. Instead, NMR analysis would have to be performed to elucidate the exact structure of the peak, however, limited sample amounts impeded further analysis so far. It could therefore not be assessed whether UGT73AH1 attaches the hexose to one of the hydroxyls or the carboxyl of asiatic acid, and the (product) regio-specificity of the enzymes remains to be determined.

To date, 33 oleanane- and ursane-type saponins have been reported in C. asiatica [26], all of them containing a glucose linked to the triterpene C-28 carboxyl, whereas no glucose linked with C-2 $\alpha$, C-3 $\beta$, and C-23 positions has been observed. Therefore, it seems very likely that UGT73AH1 catalyzes glycosylation at the $\mathrm{C}-28$ carboxyl function en route to the asiaticoside triterpene saponins.

\section{Materials and Methods}

\subsection{Plant Material and Sampling}

Whole plant cultures of $C$. asiatica were established from node segments as previously described by Kim et al. [18]. Plants generated from four independent node segments were cultured on 1/2 Murashige and Skoog (MS) liquid medium supplemented with $3 \%$ sucrose at $23 \pm 2{ }^{\circ} \mathrm{C}$ under light at $100 \mathrm{rpm}$ for 2 weeks, then $0.1 \mathrm{mM}$ methyl jasmonate (MeJA) (Sigma-Aldrich, Darmstadt, Germany) was added to the medium. Twenty-four hours after MeJA elicitation, leaves were harvested, immediately frozen in liquid nitrogen, and used for extraction of total RNA.

\subsection{EST Assembly and Annotation}

Total RNA samples of $C$. asiatica were sequenced using the Genome sequencer FLX (454 Life Sciences, Roche, Basel, Switzerland), and the raw data were processed with the Roche GS FLX software (version 2.5.3, 454 Life Sciences, Roche, Basel, Switzerland) at quality score threshold 40 and quality-trimmed with Lucy software (version 2.19; available online: http:/ /lucy.sourceforge.net/) [27] and SeqClean (version 1.0; available online: http:/ / compbio.dfci.harvard.edu). The resulting sequence reads were assembled de novo using the software package Newbler (a) set to extend low-depth overlaps. The assembled unique transcripts were first compared to the GenBank database using the BLASTN algorithm with an $E$ value cut-off of $10^{-3}$, to identify and remove ribosomal RNA sequences. The remaining sequences, putatively encoding proteins, were searched against the Arabidopsis protein database at The Arabidopsis Information Resource (TAIR; available online: http:/ / www.arabidopsis.org), the Swiss-Prot protein database (Available online: http:/ / www.expasy.ch/sprot; released on 11/20/2013), and the NCBI non-redundant protein (Nr) database (Available online: http:/ / www.ncbi.nlm.nih.gov; released on 11/20/2013) using the BLASTX algorithm with an $E$ value cut-off of $10^{-3}$. All the resulting C. asiatica high-quality reads were deposited at NCBI, and can be accessed in the Sequence Read Achieve (SRA) database under project accession number SRX1050304.

\subsection{Expression Levels of UGT Genes in C. asiatica}

Expression levels of all isotigs were calculated using the RPKM (Reads Per Kilobase per Milion mapped reads) method [28], with the formula $\operatorname{RPKM}(\mathrm{A})=(1,000,000 \times \mathrm{C}) /(\mathrm{N} \times \mathrm{L} \times 1000)$, where $\operatorname{RPKM}(\mathrm{A})$ is the expression of gene $\mathrm{A}, \mathrm{C}$ is the number of reads that uniquely align to gene $\mathrm{A}$, and $\mathrm{N}$ is the total number of bases in gene $\mathrm{A}$, and $\mathrm{L}$ is the exon length in base pairs. Statistical comparisons were performed as described by Audic and Claverie [29]. FDR (False Discovery Rate) correction was used to determine the $p$ values in multiple tests. Unigenes were considered differentially expressed when the RPKM of treated sample and control displayed a more than twofold change, with $p$ less than $10^{-3}$. 


\subsection{Phylogenetic Tree Analysis}

The protein sequences were aligned with the CLUSTALW program and phylogenetic trees constructed using the MEGA5.2 software [30] using the neighbor-joining method and bootstrapping for 1000 replicates.

\subsection{Real-Time RT-PCR Analysis}

Hairy roots of $C$. asiatica treated with MeJA for $0,12,24,48$, or $72 \mathrm{~h}$ cDNAs were used to harvest total RNA as described by Kim et al. [31]. First-strand cDNA was synthesized using the AMV reverse transcriptase (Promega, Medison, WI, USA) and $2 \mu \mathrm{g}$ of total RNA and SYBR Green Master Mix (Bio-Rad, Hercules, CA, USA) for quantification with the CFX96TM Real-Time System (Bio-Rad) and gene-specific primers (Table S2). PCR conditions were as follows: initial denaturation at $95{ }^{\circ} \mathrm{C}$ for $3 \mathrm{~min}$, followed by 40 cycles of amplification for $15 \mathrm{~s}$ at $95^{\circ} \mathrm{C}$, for $15 \mathrm{~s}$ at $58{ }^{\circ} \mathrm{C}$ and for $30 \mathrm{~s}$ at $72{ }^{\circ} \mathrm{C}$. After completing the reactions, the threshold cycle $\left(C_{t}\right)$ value for each reaction was obtained, and the differences were calculated using the delta-delta- $C_{t}$ method, with the $\beta$-actin gene (GenBank accession No. JK517508) as internal control. The fold change in transcript levels for each gene is presented as the mean and standard error of three independent experiments.

\subsection{Expression of UGT Genes}

Full-length cDNAs of target UGT were cloned using the Gateway system with specific primers, based on sequences from the transcriptome data analysis. Sequence information for the putative UGT genes has been deposited in GenBank with accession numbers (UGT73AH1, MF471454; UGT73A21, MF471455; UGT73A22, MF471459; UGT74AH1, MF471458). To minimize inclusion bodies of recombinant proteins, complete open reading frames (ORFs) with stop codon were used [25]. The ORFs were amplified by PCR from the original cDNA with attB-modified custom primers for four UGTs, and the PCR products were inserted into the $\mathrm{pDONR} /$ Zeo entry vector following the manufacturer's protocol. Plasmid DNAs were prepared from several transformants and sequenced. The E. coli expression clone was constructed using the entry vector and a destination vector pET62-DEST (Merck KGaA, Darmstadt, Germany) following the protocol provided. After confirmation of sequences, the plasmids were used for E. coli transformation.

Single colonies of BL21 (DE3) cells harboring the pET-UGT73AH1 or pET-UGT74AH1 vectors were cultured at $37^{\circ} \mathrm{C}$ in liquid lysogeny broth (LB) medium containing $50 \mu \mathrm{g} / \mathrm{mL}$ ampicillin to an optical density of 0.5 at $600 \mathrm{~nm}$. Then, the cultures were shifted to $28^{\circ} \mathrm{C}$, and isopropyl thio- $\beta$-D-galactoside (IPTG) was added to a final concentration of $0.5 \mathrm{mM}$, and incubation was continued at $20{ }^{\circ} \mathrm{C}$ for $16 \mathrm{~h}$. Finally, cells were collected by centrifugation at $15,000 \times \mathrm{g}$ for $5 \mathrm{~min}$, suspended in extraction buffer ( $50 \mathrm{mM}$ sodium phosphate, $\mathrm{pH} 7.4,300 \mathrm{mM}$ sodium chloride, $20 \%$ glycerol), and then disrupted by sonication for three times $10 \mathrm{~s}$. After cell debris was removed by centrifugation at $15,000 \times g$, the supernatant was checked for the presence of the expressed protein using 10\% SDS-PAGE. The total protein extract was used for in vitro assays, performed by incubating $1 \mathrm{mg}$ protein in $1.5 \mathrm{~mL} 50 \mathrm{mM}$ Tris- $\mathrm{HCl}, \mathrm{pH} 8.5$, containing $5 \mathrm{mM}$ uridine- $5^{\prime}$-diphosphoglucose and $0.5 \mathrm{mM}$ asiatic acid, at $35^{\circ} \mathrm{C}$ for $4 \mathrm{~h}$. Finally, $1.5 \mathrm{~mL}$ butanol was added to stop the reaction and extract products, and the organic layer was collected, and the solvent evaporated under nitrogen gas. The resulting residue was re-dissolved in $200 \mu \mathrm{L}$ of methanol, and stored at $4{ }^{\circ} \mathrm{C}$ for chemical analysis.

\subsection{UPLC-QTOF/MS Analysis of the Products from Enzymatic Reactions}

UPLC was performed using an ACQUITY H-Class UPLC (Waters, Milford, MA, USA) according to the protocol reported by Lee et al. [32] with an ACQUITY BEH C18 column $(2.1 \mathrm{~mm} \times 100 \mathrm{~mm}$, $1.7 \mu \mathrm{m}$ ) held at $40{ }^{\circ} \mathrm{C}$. Two microliters of sample were injected for separation, with a mobile phase consisting of Solvent A ( $5 \%$ acetonitrile, $0.1 \%$ formic acid) and Solvent B ( $95 \%$ acetonitrile, $0.1 \%$ formic acid) at a constant flow rate of $450 \mu \mathrm{L} / \mathrm{min}$. Eluting compounds were detected with a Waters Xevo 
G2-S QTOF-MS (Waters) operating in positive and negative ion mode, with alternating high- and low-energy scans (MSE acquisition mode) and cone voltage $40 \mathrm{~V}$, capillary voltage $3.0 \mathrm{kV}$, source temperature $120^{\circ} \mathrm{C}$, desolvation temperature $300^{\circ} \mathrm{C}$, cone gas flow $30 \mathrm{~L} / \mathrm{h}$, and desolvation gas flow $600 \mathrm{~L} / \mathrm{h}$. Accurate mass measurements were obtained by means of an automated calibration delivery system using leucine as internal reference $\left(m / z 556.276\left(\mathrm{ESI}^{+}\right), m / z 554.262\left(\mathrm{ESI}^{-}\right)\right)$. Data were collected between $m / z 100$ and $m / z 2000$.

\section{Conclusions}

Four candidate genes of UGT were selected from transcriptome data based sequence comparisons, and based on their expression patterns in MeJA-elicited C. asiatica leaves. UPLC-TOF/MS analysis of in vitro assays showed that UGT73AH1 produces asiatic acid monohexoside. We thus demonstrated that the UGT73AH1 protein catalyzes the glycosylation of a ursane-type triterpene, now enabling further investigations into the genetic engineering of triterpene saponin biosynthesis. As part of these studies, it will be interesting to test the relative activity of the enzyme on other substrates, including madecassic acid as well as oleanane backbone isomers of both asiatic and madecassic acids.

Supplementary Materials: Supplementary materials can be found at www.mdpi.com/1422-0067/18/12/2630/s1.

Acknowledgments: We thank Mackenzie for help with the UGT nomenclature. This work was supported by the "International Cooperative Research Program for Agricultural Science \& Technology Development (Project No. PJ00849903)", RDA, Korea.

Author Contributions: Ok Tae Kim performed most of the experiments, analyzed data, and drafted the manuscript. Mei Lan Jin took part in molecular works and Dae Young Lee performed the UPLC-QTOF/MS analysis. Reinhard Jetter revised the manuscript. All authors read and approved the manuscript.

Conflicts of Interest: The authors declare no conflict of interest.

\section{Abbreviations}

$\begin{array}{ll}\text { UGT } & \text { UDP-glucosyltransferase } \\ \text { MeJA } & \text { Methyl jasmonate } \\ \text { RPKM } & \text { Reads per kilobase per million mapped reads } \\ \text { EST } & \text { Expressed sequence tag } \\ \text { UPLC-QTOP/MS } & \begin{array}{l}\text { Ultra-high Performance Liquid Chromatography-Quadrupole Time-of-Flight } \\ \text { Mass Spectrometry }\end{array}\end{array}$

\section{References}

1. Jones, P.; Vogt, T. Glycosyltransferases in secondary plant metabolism: Tranquilizers and stimulant controllers. Planta 2001, 213, 164-174. [CrossRef] [PubMed]

2. Vogt, T.; Jones, P. Glycosyltransferases in plant natural product synthesis: Characterization of a supergene family. Trends Plant Sci. 2000, 5, 380-386. [CrossRef]

3. Phillips, D.R.; Rasbery, J.M.; Bartel, B.; Matsuda, S.P.T. Biosynthetic diversity in plant triterpene cyclization. Curr. Opin. Plant Biol. 2006, 9, 305-314. [CrossRef] [PubMed]

4. Seki, H.; Tamura, K.; Muranaka, T. P450s and UGTs: Key players in the structural diversity of triterpenoid saponins. Plant Cell Physiol. 2015, 56, 1463-1471. [CrossRef] [PubMed]

5. Thimmappa, R.; Geisler, K.; Louveau, T.; O'Maille, P.; Osbourn, A. Triterpene biosynthesis in plants. Annu. Rev. Plant Biol. 2014, 65, 225-257. [CrossRef] [PubMed]

6. Xu, R.; Fazio, G.C.; Matsuda, S.P.T. On the origins of triterpenoid skeletal diversity. Phytochemistry 2014, 65, 261-291. [CrossRef]

7. Ross, J.; Li, Y.; Lim, E.K.; Bowles, D.J. Higher plant glycosyltransferases. Genome Biol. 2001, 2, 3004.1-3004.6. [CrossRef]

8. Li, Y.; Baldauf, S.; Lim, E.K.; Bowles, D.J. Phylogenetic analysis of the UDP-glycosyltransferase multigene family of Arabidopsis thaliana. J. Biol. Chem. 2001, 276, 4338-4343. [CrossRef] [PubMed] 
9. Achnine, L.; Huhman, D.V.; Farag, M.A.; Sumner, L.W.; Blount, J.W.; Dixon, R.A. Genomics-based selection and functional characterization of triterpene glycosyltransferases from the model legume Medicago truncatula. Plant J. 2005, 41, 875-887. [CrossRef] [PubMed]

10. Paquette, S.; Møller, B.L.; Bak, S. On the origin of family 1 plant glycosyltransferases. Phytochemistry 2003, 62, 399-413. [CrossRef]

11. Yonekura-Sakakibara, K.; Hanada, K. An evolutionary view of functional diversity in family 1 glycosyltransferases. Plant J. 2011, 66, 182-193. [CrossRef] [PubMed]

12. Shibuya, M.; Nishimura, K.; Yasuyama, N.; Ebizuka, Y. Identification and characterization of glycosyltransferases involved in the biosynthesis of soyasaponin I in Glycine max. FEBS Lett. 2010, 584, 2258-2264. [CrossRef] [PubMed]

13. Owatworakit, A.; Townsend, B.; Louveau, T.; Jenner, H.; Rejzek, M.; Hughes, R.K.; Saalbach, G.; Qi, X.; Bakht, S.; Roy, A.D.; et al. Glycosyltransferases from oat (Avena) implicated in the acylation of avenacins. J. Biol. Chem. 2013, 288, 3696-3704. [CrossRef] [PubMed]

14. Naoumkina, M.A.; Modolo, L.V.; Huhman, D.V.; Urbanczyk-Wochniak, E.; Tang, Y.; Sumner, L.W.; Dixon, R.A. Genomic and coexpression analyses predict multiple genes involved in triterpene saponin biosynthesis in Medicago truncatula. Plant Cell 2010, 22, 850-866. [CrossRef] [PubMed]

15. Meesapyodsuk, D.; Balsevich, J.; Reed, D.W.; Covello, P.S. Saponin biosynthesis in Saponaria vaccaria. cDNAs encoding beta-amyrin synthase and a triterpene carboxylic acid glucosyltransferase. Plant Physiol. 2007, 143, 959-969. [CrossRef] [PubMed]

16. Augustin, J.M.; Drok, S.; Shinoda, T.; Sanmiya, K.; Nielsen, J.K.; Khakimov, B.; Olsen, C.E.; Hansen, E.H.; Kuzina, V.; Ekstrøm, C.T.; et al. UDP-glycosyltransferases from the UGT73C subfamily in Barbarea vulgaris catalyze sapogenin 3-O-glucosylation in saponin-mediated insect resistance. Plant Physiol. 2012, 160, 1881-1895. [CrossRef] [PubMed]

17. De Costa, F.; Barber, C.J.S.; Kim, Y.B.; Reed, D.W.; Zhang, H.; Fett-Neto, A.; Covello, P.S. Molecular cloning of an ester-forming triterpenoid: UDP-glucose 28-O-glucosyltransferase involved in saponin biosynthesis from the medicinal plant Centella asiatica. Plant Sci. 2017, 262, 9-17. [CrossRef] [PubMed]

18. Kim, O.T.; Kim, M.Y.; Hong, M.H.; Ahn, J.C.; Hwang, B. Stimulation of asiaticoside production from Centella asiatica whole plant cultures by elicitors. Plant Cell Rep. 2004, 23, 339-344. [CrossRef] [PubMed]

19. James, J.T.; Dubery, I.A. Pentacyclic triterpenoids from the medicinal herb, Centella asiatica (L.) Urban. Molecules 2009, 14, 3922-3941. [CrossRef] [PubMed]

20. Park, J.H.; Choi, J.Y.; Son, D.J.; Park, E.K.; Song, M.J.; Hellström, M.; Hong, J.T. Anti-inflammatory effect of titrated extract of Centella asiatica in phthalic anhydride-induced allergic dermatitis animal model. Int. J. Mol. Sci. 2017, 18, 738. [CrossRef] [PubMed]

21. Pittella, F.; Dutra, R.C.; Junior, D.D.; Lopes, M.T.; Barbosa, N.R. Antioxidant and cytotoxic activities of Centella asiatica (L) Urb. Int. J. Mol. Sci. 2009, 10, 3713-3721. [CrossRef] [PubMed]

22. Matsuda, H.; Morikawa, T.; Ueda, H.; Yoshikawa, M. Medicinal Foodstuffs. XXVII. Saponin constituents Gotu Kola (2): Structures of new ursane- and oleanane-type triterpene oligoglycosides, centellasaponin B, C, and D, from Centella asiatica cultivated in Sri Lanka. Chem. Pharm. Bull. 2001, 49, 1368-1371. [CrossRef] [PubMed]

23. Sangwan, R.S.; Tripathi, S.; Singh, J.; Narnoliya, L.K.; Sangwan, N.S. De novo sequencing and assembly of Centella asiatica leaf transcriptome for mapping of structural, functional and regulatory genes with special reference to secondary metabolism. Gene 2013, 525, 58-76. [CrossRef] [PubMed]

24. Mackenzie, P.I.; Owens, I.S.; Burchell, B.; Bock, K.W.; Bairoch, A.; Bélanger, A.; Fournel-Gigleux, S.; Green, M.; Hum, D.W.; Iyanagi, T.; et al. The UDP glycosyltransferase gene superfamily: Recommended nomenclature update based on evolutionary divergence. Pharmacogenetics 1997, 7, 255-269. [CrossRef] [PubMed]

25. Zhu, S.; Gong, C.; Ren, L.; Li, X.; Song, D.; Zheng, G. A simple and effective strategy for solving the problem of inclusion bodies in recombinant protein technology: His-tag deletions enhance soluble expression. Appl. Microbiol. Biotechnol. 2013, 97, 837-845. [CrossRef] [PubMed]

26. Azerad, R. Chemical structures, production and enzymatic transformations of sapogenins and saponins from Centella asiatica (L.) Urban. Fitoterapia 2016, 114, 168-187. [CrossRef] [PubMed]

27. Chou, H.H.; Holmes, M.H. DNA sequence quality trimming and vector removal. Bioinfomatics 2001, 17, 1093-1104. [CrossRef] 
28. Mortazavi, A.; Williams, B.A.; McCue, K.; Schaeffer, L.; Wold, B. Mapping and quantifying mammalian transcriptomes by RNA-Seq. Nat. Methods 2008, 5, 621-628. [CrossRef] [PubMed]

29. Audic, S.; Claverie, J.M. The significance of digital gene expression profiles. Genome Res. 1997, 7, 986-995. [CrossRef] [PubMed]

30. Tamura, K.; Peterson, D.; Peterson, N.; Stecher, G.; Nei, M.; Kumar, S. MEGA5: Molecular evolutionary genetics analysis using maximum likelihood, evolutionary distance, and maximum parsimony methods. Mol. Biol. Evol. 2011, 28, 2731-2739. [CrossRef] [PubMed]

31. Kim, O.T.; Bang, K.H.; Shin, Y.S.; Lee, M.J.; Jung, S.J.; Hyun, D.Y.; Kim, Y.C.; Seong, N.S.; Cha, S.W.; Hwang, B. Enhanced production of asiaticoside from hairy root cultures of Centella asiatica (L.) Urban elicited by methyl jasmonate. Plant Cell Rep. 2007, 26, 1941-1949. [CrossRef] [PubMed]

32. Lee, J.W.; Ji, S.H.; Kim, G.S.; Song, K.S.; Um, Y.; Kim, O.T.; Lee, Y.; Hong, C.P.; Shin, D.H.; Kim, C.K.; et al. Global Profiling of Various Metabolites in Platycodon grandiflorum by UPLC-QTOF/MS. Int. J. Mol. Sci. 2015, 16, 26786-26796. [CrossRef] [PubMed]

(C) 2017 by the authors. Licensee MDPI, Basel, Switzerland. This article is an open access article distributed under the terms and conditions of the Creative Commons Attribution (CC BY) license (http://creativecommons.org/licenses/by/4.0/). 